\title{
The waves of damage in elastic-plastic lattices with waiting links: design and simulation
}

\author{
A. Cherkaev *, V. Vinogradov, S. Leelavanichkul \\ Department of Mathematics, University of Utah, 155 S 1400 E, Salt Lake City, \\ UT 84112, USA
}

\begin{abstract}
We consider protective structures with elastic-plastic links of a special morphology. The links are bistable: they are designed to arrest the development of localized damage (a neck) in an element, initiating the damage instead in another sequential element. A wave of "partial damage" propagates through the chain, as all the links develop necks but do not fail. When subjected to an impact, such a structure absorbs several times more energy than a conventional structure, which makes it more protective against collision. A three-dimensional finite element analysis is used to compute the force-elongation relation for a bistable element. Another numerical procedure analyzes the dynamics of a chain or a lattice undergoing a collision with a heavy projectile. The procedure describes the nonlinear waves, determines the places of failure and the critical loading conditions. A bistable chain is compared with a chain of conventional links, of the same length, mass, and material. The bistable chain is capable to withstand a collision with a mass of kinetic energy, several times greater. Even when the bistable chain breaks it is able to more effectively reduce the speed of the projectile.
\end{abstract}

Key words: Protective structure, Elastic-plastic material, Necking, Bistability, Partial damage, Collision, Waves of transition, Failure.

\section{Introduction}

The failure of an elongated specimen occurs when a localized damaged zone expands across the sample while the large part of the material of a broken sample stays undamaged. It is tempting to design the specimen that uniformly distributes a "partial

* Corresponding author.

Email addresses: cherk@math.utah.edu (A. Cherkaev), vladim@math.utah.edu (V. Vinogradov), sleela@math.utah.edu (S. Leelavanichkul). 
damage" in its volume and utilizes all the material capability. Such a specimen absorbs more energy before failure that goes to develop the damage. The problem is to create a structure capable to distribute a damage and absorb the energy of an impact without failure.

The difficulty is the natural localization of the damage: In solid bars, the damaged cross-section is weaker and the material in it is more vulnerable to failure than the material in the undamaged parts. The maximal stress in solid material is always located near the zones of impact and support. A more uniform distribution of the stress can be achieved in a structure with bistable links. Such a structure experiences a phase transition: The links transit from initial undamaged state to the partial damaged state. These two locally stable states of a link are separated by an interval of unstable deformation. The transition of a link causes the transition of a neighboring link which initiates a nonlinear wave of damage that is absorbed by the structure. We investigate structures with artificially induced instabilities due to use of links of special morphology - the waiting links which possess nonmonotonic constitutive relations. Previously, the waiting link structures were investigated in a number of papers. The concept of the structures with waiting links were suggested in (1). The dynamics of reversible bistable structures were addressed in $(6 ; 7)$. The waves in elastic-brittle structures were studied in $(2 ; 3 ; 4 ; 8)$. The theory of the waves in unstable materials is considered in the book $(9)$ and in the papers $(10 ; 11 ; 12)$. The dynamics of lattices were simulated in (5).

In this paper, we deal with bistable structures from the elastic-plastic materials. The model is more sophisticated than the elastic-brittle models that were examined before. The ANSYS simulation is used to obtain the constitutive relations in a bistable link; the model accounts for the hysteresis of the loading cycle, the realistic geometric parameters, elastic-plastic properties of the specific material, etc. The two simulations are used: The first one results in a static constitutive equations and the second in the dynamics of the phase transition and damage propagation. Each link of the assembly possesses a nonmonotonic force-elongation relation; the link can experience an irreversible damage (partial failure) after which its equilibrium length increases and elastic modulus changes. It turns out that the waiting-link-structures sustain a much larger impact than the conventional ones due to the distribution of the partial damage and the increase of the related energy release. The expected applications range from hypersensitive sensors that change their properties in response to a signal, to protective structures, to "dynamic smart materials," to the controllable damage propagation. 


\section{Structures with waiting links}

\subsection{Mechanism of damage propagation}

Consider a chain or lattice of bistable links from an elastic-plastic material. Each bistable link consists of the shorter main link and the elongated and initially curved waiting link. The main and waiting links are made of the same elastic-plastic material and joined at the ends. An example of a one-dimensional chain is shown in Figure 1.

Initially, the resistance of the assembly is mainly due to the elastic response of the main link; the resistance of the bended waiting link is relatively small. As the elongation increases, the main link enters the plastic zone and develops a neck which reduces its resistance. At that moment, the waiting link is straightened and its resistance sharply increases. The sum of resistances of the two links becomes bistable, if the parameters of these links are properly chosen: The total resistance increases, then decreases, then increases again. The increasing regimes correspond to the locally stable state, and the decreasing regime to the unstable state. The passage between these states is the phase transition.

The properly designed bistable chain is much more resistant than the conventional chain. In a conventional elongated bar of elastic-plastic material, the necking corresponds to the beginning of a fatal instability; the damage is accumulated in the region of the neck and the sample breaks. Only one neck is developed in the sample independently of its length. In a bistable assembly, the activated waiting link arrests the development of the irreversible plastic deformation and prevents the failure of the necked main link. When the elongation of the whole chain increases, another neck is formed in a neighbor assembly instead, and the damage propagates. This sequentially occurred transformation from one stable state to another creates a transition wave which propagates along the chain. The process continues until all links experience

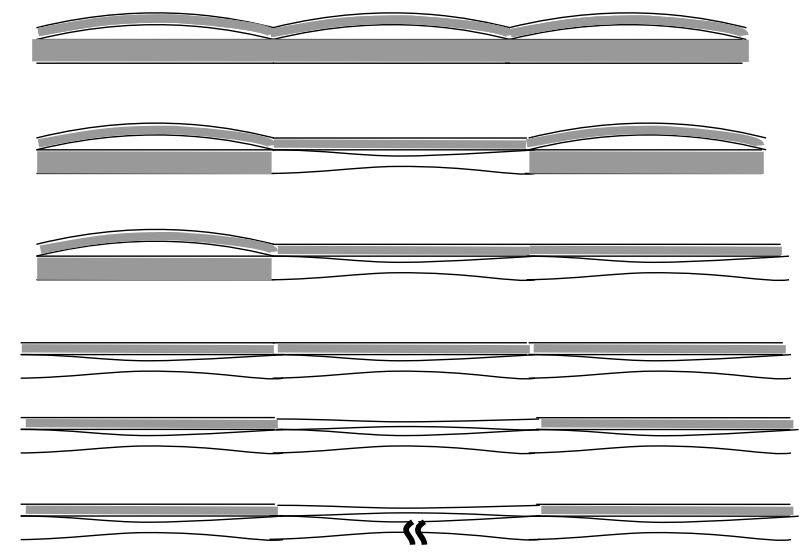

Fig. 1. Progressive damage of elastic-plastic chain under tensile loading. The grey elements are undamaged, the white elements are partially damaged. 
the transition; then, the deformation in the waiting links also become plastic and the chain breaks.

Notice that the elongation of a rectangular lattice under plain normal impact is equivalent to the elongation of the chain (Figure 2). The links that are perpendicular to the loading are not stressed. They enter the equations only by addition of their mass at the joints. The static behavior is analogous to the behavior of a chain.

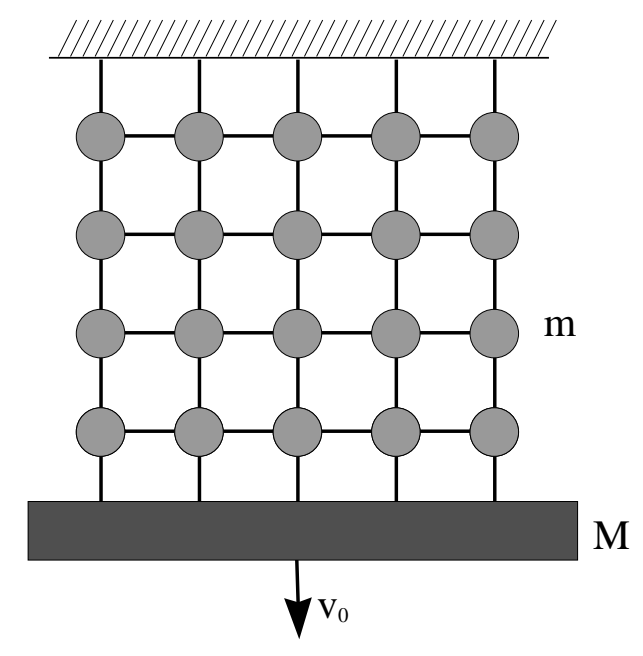

Fig. 2. Structure of the rectangular lattice

Compared with the elastic-brittle lattices investigated earlier, the elastic-plastic protective structures naturally survive larger deformations, which increases the failure limit and the relaxation capabilities. The waiting link may be made considerably thinner in comparison to the main link, because its role is to stop the fatal instability of the neck. To the contrary, the structure with elastic-brittle elements will survive the failure of a main link only if the waiting link is thicker than the main one.

\subsection{Simulation of the bistable link}

The finite element simulation is used to demonstrate the bistable force-displacement relation. Copper C26000 was chosen because of its low hardening and high ductility. Geometrically, the assembly consists of the main link $(25.4 \mathrm{~mm} . \times 7.94 \mathrm{~mm}$. $\times$ $1.59 \mathrm{~mm}$.$) in the center and two equal waiting links (27.94 \mathrm{~mm} . \times 6.35 \mathrm{~mm} . \times .79 \mathrm{~mm}$. at the top and bottom, joined at the ends. This design provides a symmetric deformation of the main link.

The simulations are carried out using ANSYS 8.0. In a simulated model of the bistable link, the geometric and material nonlinearities and finite deformations are taken into account. The 3-D model is used in order to correctly simulate necking. The finite element simulation is performed using the 8-node element having three degrees of 

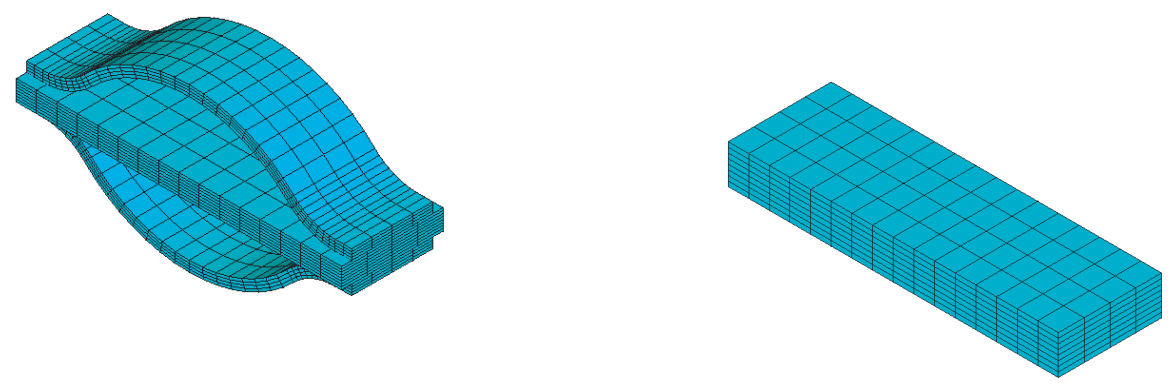

Fig. 3. Meshed models for the bistable and solid links

freedom at each node (SOLID45). The meshed model of the structure is shown in Figure 3. Finer mesh resolution does not yield a noticeable differences in the results.

To simulate the tensile experiment, the displacement is applied in the longitudinal direction at both ends. The symmetry is used and the simulation is performed on one fourth of the structure. The elastic-plastic response of the assembly is simulated using von Mises yield criterion, associated flow rule, and isotropic work hardening. The computed deformation of the structure is shown in the plot of the von Mises stress (Figure 4) at the final load step. For better visibility, the second contour plot represents only a quarter of the structure. Notice that the neck in the main link is developed and arrested when the waiting link is straightened. Figure 5 shows the computed forcedisplacement relation for the main and waiting link. The force-displacement relation of the whole assembly shown in Figure 6 is obtained by summing the resistance force in the main and waiting links.

The bistable link is compared with the solid link of the same length, mass, and material, see Figure 3. The solid link also develops a neck, see Figure 7, but lacks the second stable interval of elongation. The force-elongation dependence of the solid link is shown at Figure 8.

\section{Dynamics of a chain}

\subsection{Assumptions and equations}

Addressing the dynamics of bistable chains (lattices), we use a simplified model. We assume that

(1) The simulated force-elongation dependences in the bistable and solid links are 

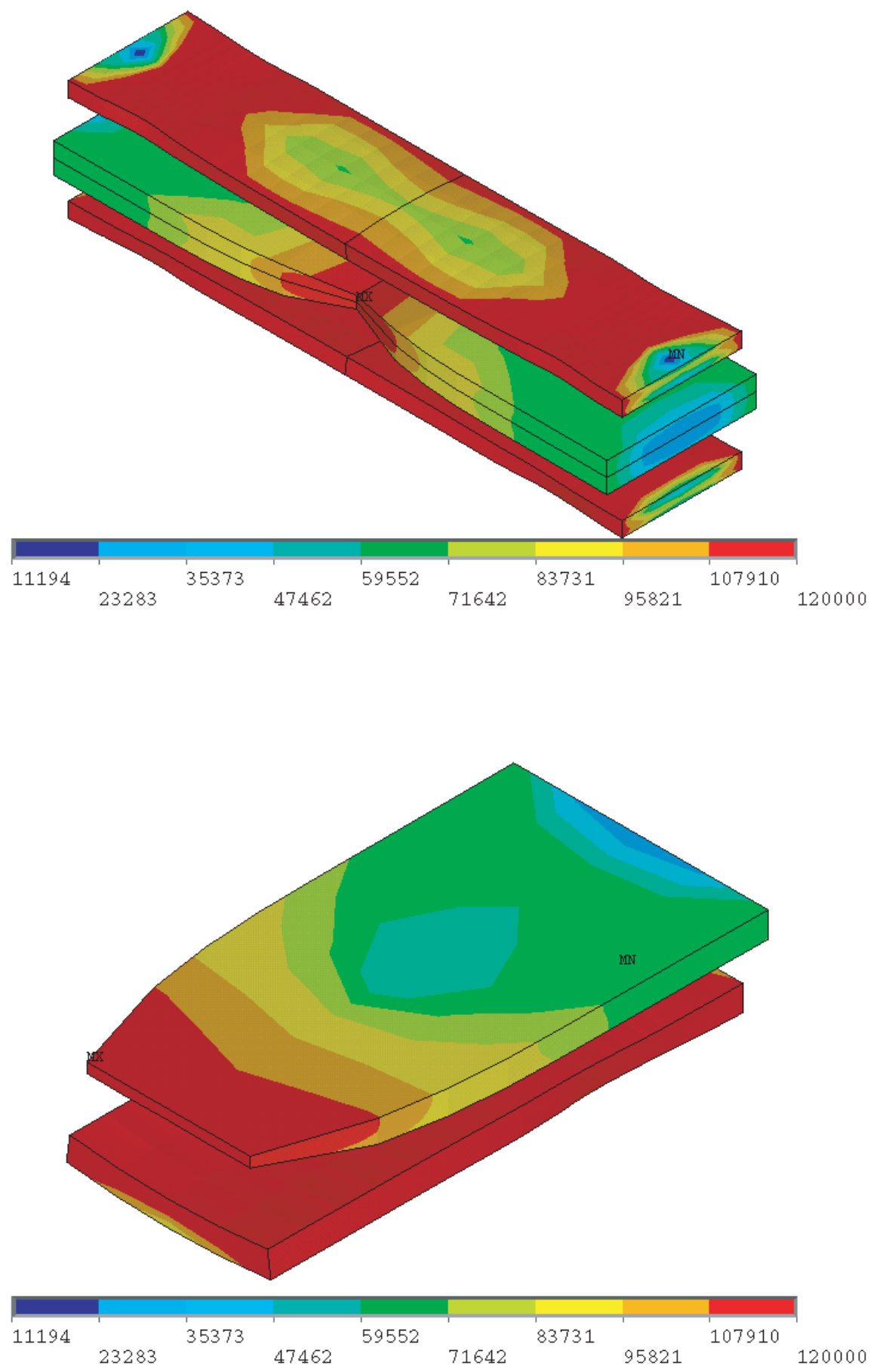

Fig. 4. Bistable link: Contour plot of von Mises stress at the final load step 


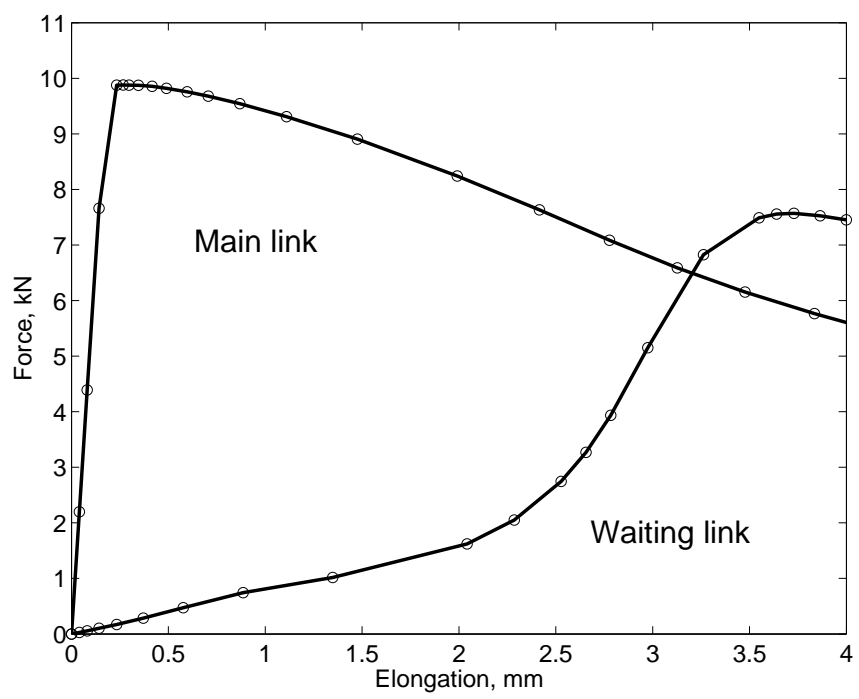

Fig. 5. Force-displacement diagram for the main and waiting links. Notice the large interval of the decreasing force in the main link due to the necking. The S-shaped diagram of the initially curved waiting link is due to its straightening.

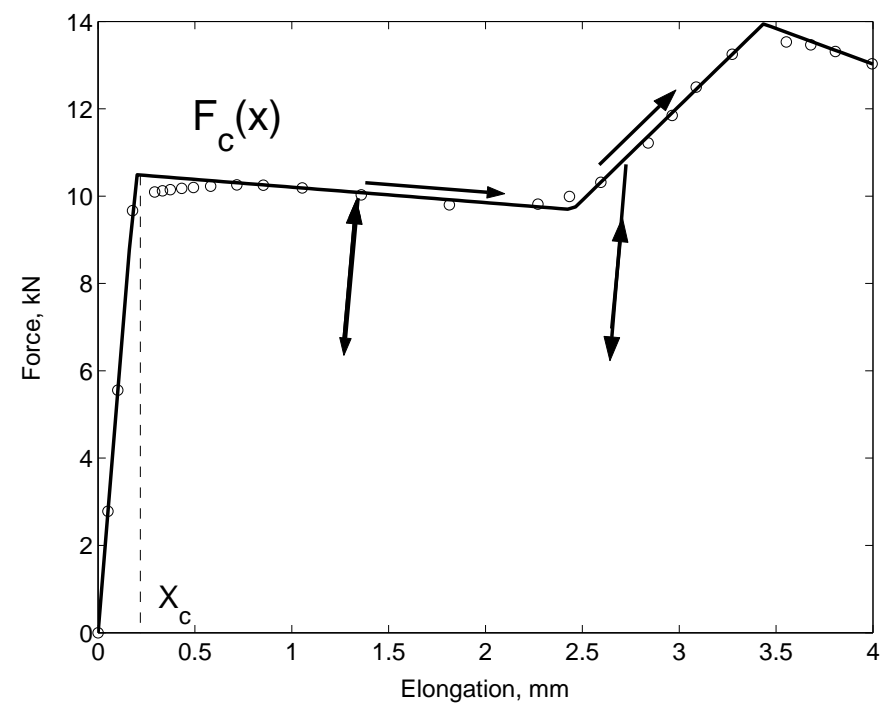

Fig. 6. Force-displacement diagram of a bistable link and its piece-wise linear approximation. replaced by the piece-wise linear functions, see Figures 6 and 8 . The figures show that the approximation is accurate; it allows for accelerating of the computations.

(2) The model separates the concentrated masses and massless springs. The mass of a whole link is concentrated in the joints between them. This assumption greatly simplifies the calculation because the chain is replaced by a mass-spring system. For the lattice model (Figure 2), the additional mass of perpendicular unstressed links is added to the mass of the stressed links at the joints.

(3) The simulated force-elongation dependence corresponds to the static response of the material. It is assumed that the same dependence describes the response to a dynamic loading. The interval of the velocities of the projectile used in the calculations does not exceed $1.2 \%$ of the sound speed in the material which 


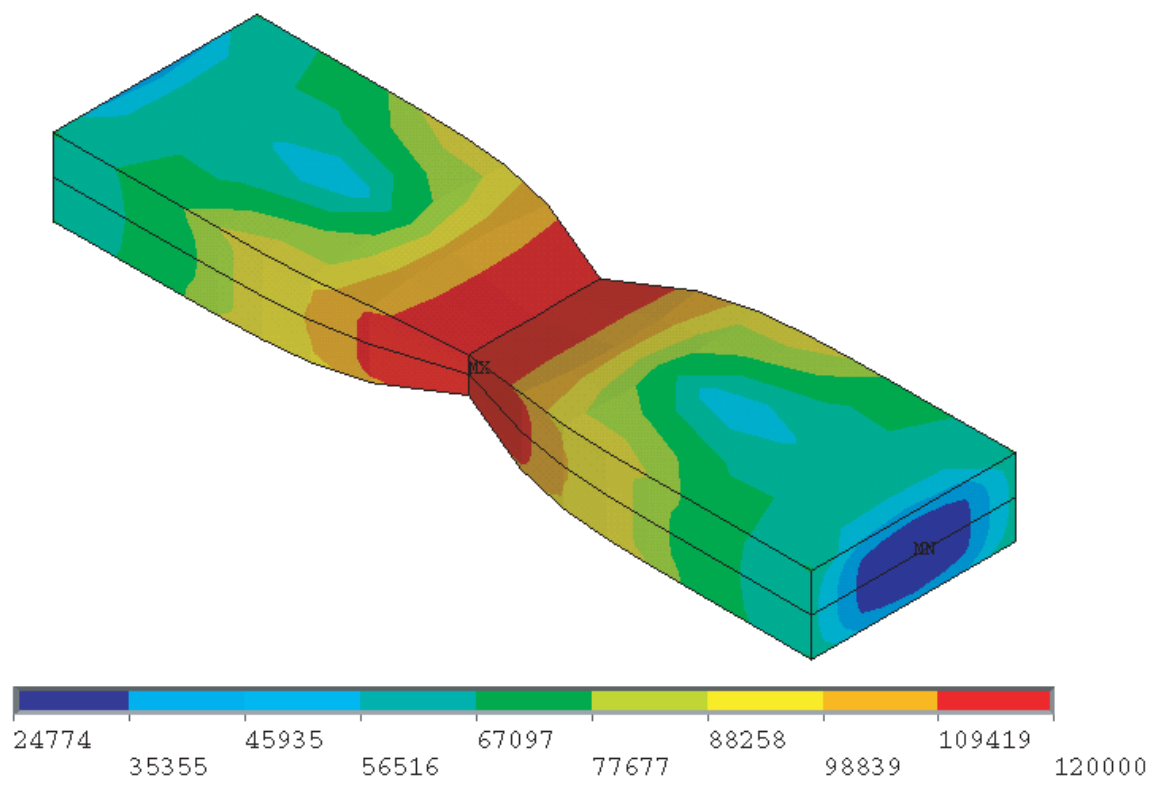

Fig. 7. Solid link: Contour plot of von Mises stress at the final load step

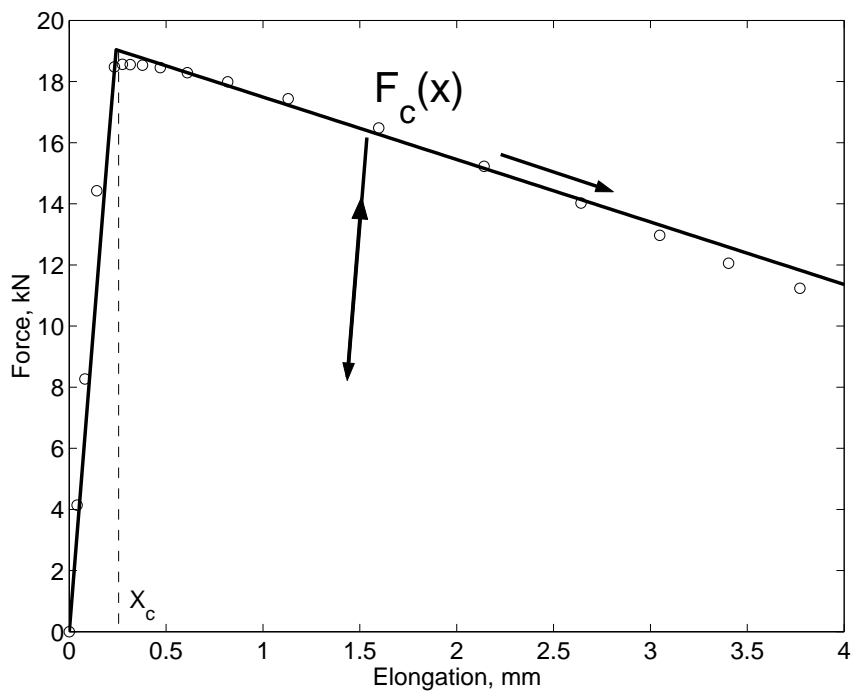

Fig. 8. Force-displacement diagram in the solid link and its piece-wise linear approximation justifies this assumption.

(4) The unloading of the elastic-plastic link follows the straight path parallel to the linear (elastic) part of the force-elongation diagram.

The elastic-plastic deformation of the link is described by the equation (for detailed discussion of the plastic flow, see $(13 ; 14)$ ) 


$$
\begin{array}{r}
F(t, x, \dot{x})=F_{c}\left(x_{p l}(t)+x_{c}\right)-c\left(x_{p l}(t)+x_{c}-x(t)\right) \\
\dot{x}_{p l}(t)=\left\{\begin{array}{llr}
0 & \text { if } x \leq x_{c} & \text { or } F<F_{c} \\
0 & \text { if } F=F_{c}, & \dot{x}(t) \leq 0 \\
\dot{x}(t) & \text { if } F=F_{c}, & \dot{x}(t)>0
\end{array}\right.
\end{array}
$$

where $x$ is the elongation of the link, $x_{c}$ is the elongation of the elastic limit, $x_{p l}$ is the plastic (irreversible) elongation, $c$ is the elastic constant. The critical forces $F_{c}(x)$ for the bistable and solid links are shown in Figures 6 and 8, respectively. They are piece-wise linear functions of $x$. Notice that $F_{c}(x)=0$ if the elongation exceeds a threshold, which corresponds to the failure. The initial condition

$$
x_{p l}(0)=0
$$

assumes that initially there is no plastic deformation.

We perform the following numeric experiment. A chain is fixed at one end and impacted by the projectile of mass $M$ at the other end. After the impact, the mass $M$ stays attached to the mass $m$ at the free end of the chain. The dynamics of the chain is described by the set of differential equations

$$
\begin{aligned}
m \ddot{z}_{n} & =F\left(t, x_{n}, \dot{x}_{n}\right)-F\left(t, x_{n-1}, \dot{x}_{n-1}\right), \\
x_{n} & =z_{n+1}-z_{n}, \quad n=1, \ldots N-1 \\
(M+m) \ddot{z}_{N} & =-F\left(t, x_{n-1}, \dot{x}_{n-1}\right) \quad z_{0}=0,
\end{aligned}
$$

where $z_{n}$ is the position of $n$th knot, $x_{n}$ is the elongation of $n$th link, $N$ is the number of links in the chain, and $z_{0}$ is the position of the fixed end of the chain. Mass $m$ of the knot is equal to the mass of the link. In the lattice model, the mass $m$ is equal to the double mass of the link; this way we account for the mass of the unstressed links in the lattice. This system is integrated with the initial conditions

$$
\begin{aligned}
& z_{n}=n a \quad \dot{z}_{n}=0, \quad n=1, \ldots N-1, \\
& z_{N}=N a, \quad \dot{z}_{N}=\frac{M}{M+m} v_{0},
\end{aligned}
$$

where $a$ is the length of the unstressed link, and $v_{0}$ is the speed of the impact. This impact delivers the impulse $M v_{0}$ to the last mass in the chain. The impulse corresponds to the initial speed $\frac{M}{M+m} v_{0}$ of that mass and the attached mass of the projectile. 

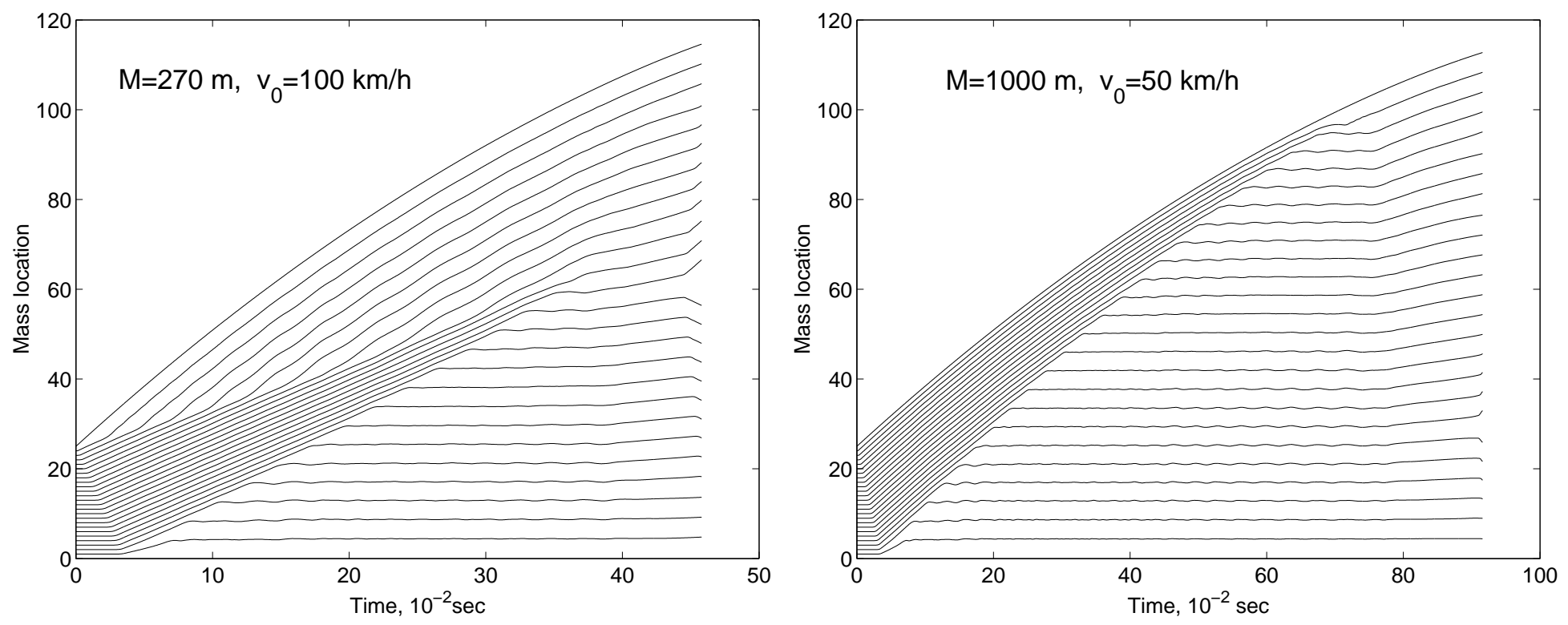

Fig. 9. Waves in elastic-plastic chain of 25 bistable links. Left: Observe the fast sonic wave, two waves of transition (damage) and the breakage when they meet. Right: The fast sonic wave initiates one transition wave.

\section{Simulation and results}

\subsection{Waves of transition}

The system of differential equations is solved using MATLAB. The typical picture of the motion of the masses in a bistable chain is shown in Figure 9. One can see that all the links develop a plastic deformation and each link develops a neck before the chain fails.

The impact initiates an elastic wave which propagates along the chain. When it reflects from the support, the amplitude of the wave doubles. If the magnitude of the wave is large enough, it transfers the first link into the plastic unstable zone. After this, the wave of the transition is initiated and propagates toward the impacted end. Simultaneously, the impact originates another transitional wave which propagates toward the support. The chain breaks when these two waves meet.

If the speed of the impact is larger, the chain fails immediately after the impact when the first link breaks, as in Figure 10. If the speed of the impact is smaller, the transition waves do not break the chain when they meet. The even smaller speed correspond to absence of one of the transitional waves, see Figure 9 or both of them. This is compared to the waves in a chain of the conventional links where transitional waves do not occur, see Figure 10. The elastic wave from the impact propagates along the chain. When it reflects from the support, it transfers the first link into the plastic unstable zone and breaks it. If the magnitude of the wave is smaller, the chain stays 

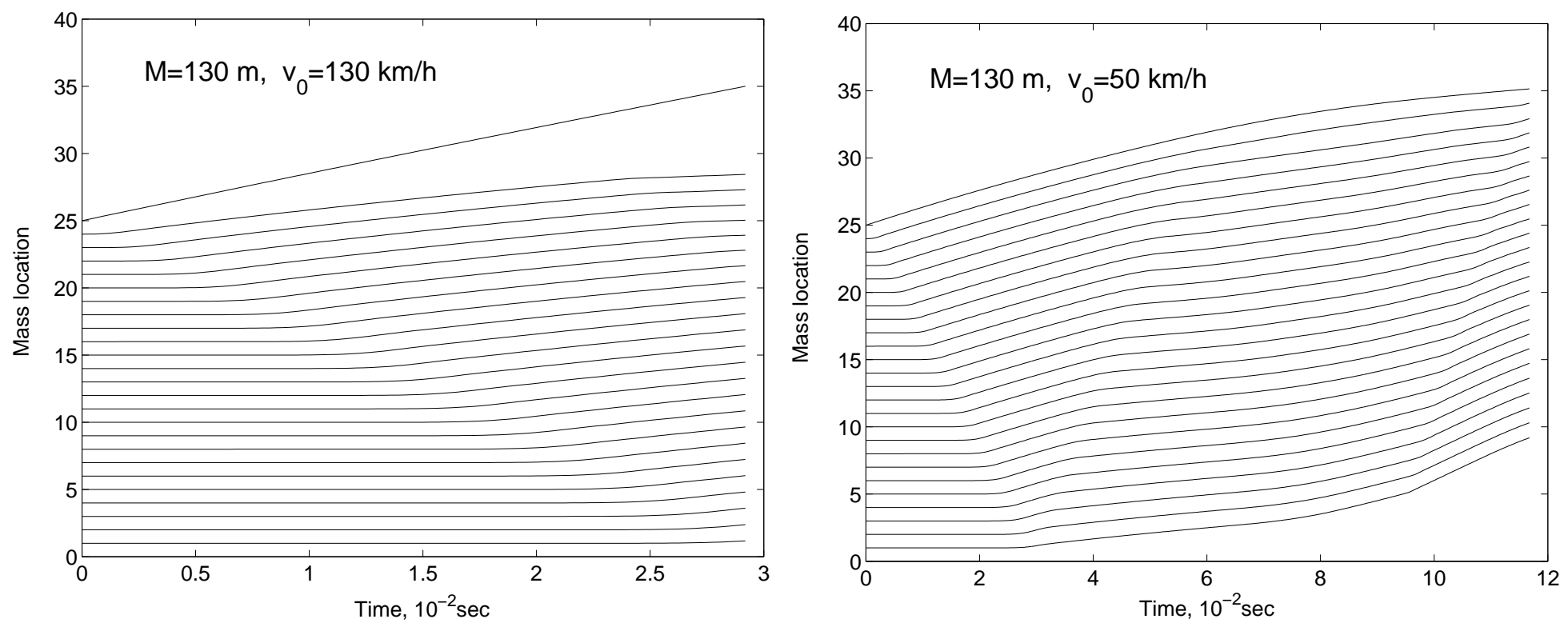

Fig. 10. Waves in elastic-plastic chain of 25 solid links. The first or the last links fail.

unbroken. The impact of a larger speed breaks the link closest to the impact.

\subsection{Energy absorption}

All the links of the broken solid chain but one are undamaged: their plastic deformation is zero. Contrary to this, the links in the bistable broken chain experience a partial damage: Each of the main links develops a neck and is plastically deformed. Therefore, the bistable chain absorbs larger energy before breakage which is uniformly distributed along its length in all partially damaged links. The bistability leads to a paradoxical result: the maximal absorbed energy in the bistable chain is proportional to its volume, not to the cross-section as in the conventional chain, as it is illustrated in Figure 11

Figure 12 compares the resistance of the bistable and conventional chains. The picture shows the kinetic energy of the projectile that breaks the chain; the energy is computed at the instance of the breakage. This energy is zero if the projectile is stopped.

The bistable chain of the same mass and length is able to capture faster projectiles. Even if it is broken, the projectile has lower residual speed when the chains break. When the speed of the projectile is very large, the both chains are broken by the projectile of the same energy; in that range, the link closest to the impact is broken and the other links do not contribute. 


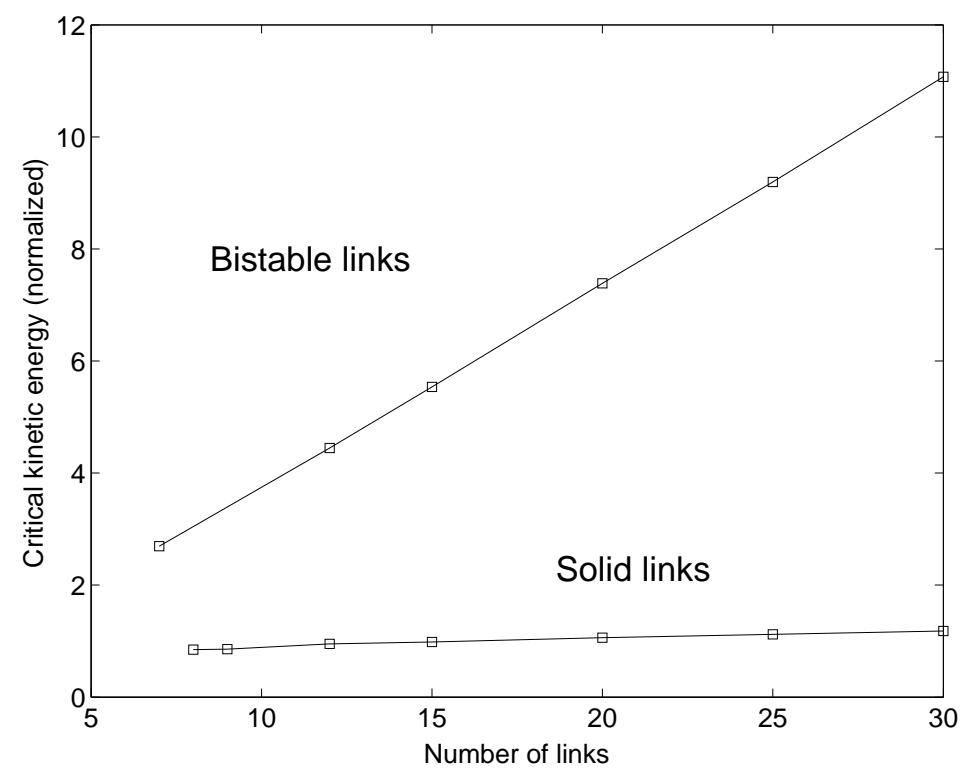

Fig. 11. Kinetic energy needed to break the bistable and conventional chains versus the number of links.

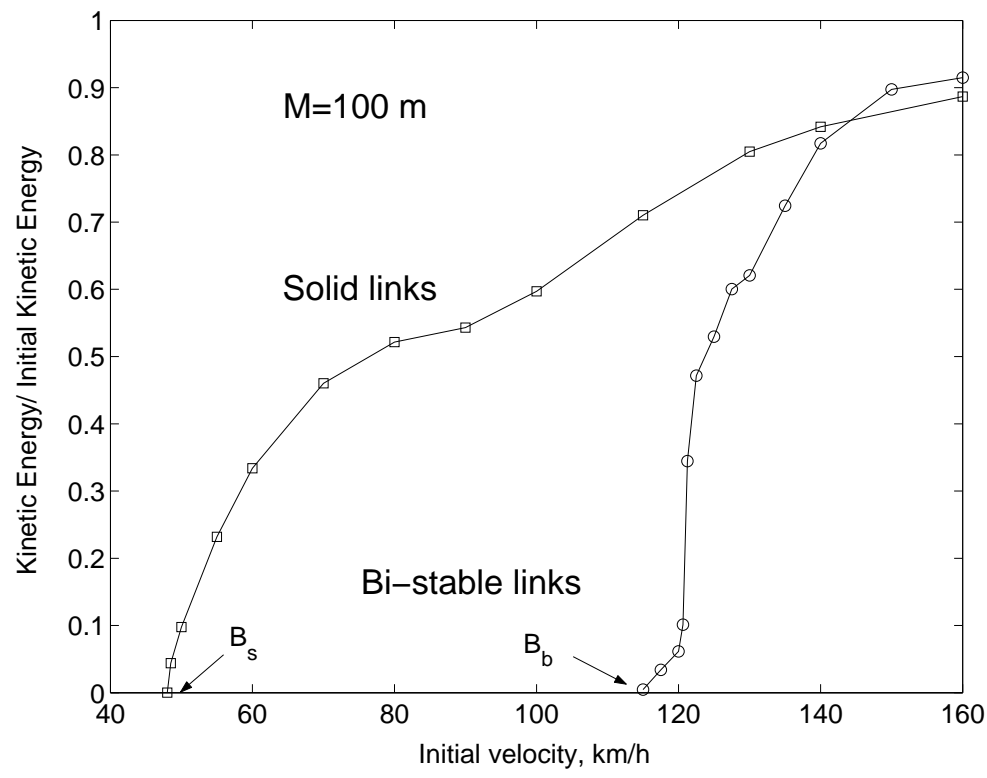

Fig. 12. Ratio of the kinetic energies of the projectile before and after collision with the chain of 15 links. Zero corresponds to captured projectile.

\subsection{Critical loading}

The points $B_{s}$ and $B_{b}$ shown in Figure 12 correspond to the critical behavior of the projectile: It breaks the chain but its speed is reduced to almost zero at that instance. If the speed of the projectile is smaller, it reverses its direction and starts to oscillate.

We call a projectile critical if it is stopped by the chain while any projectile with 


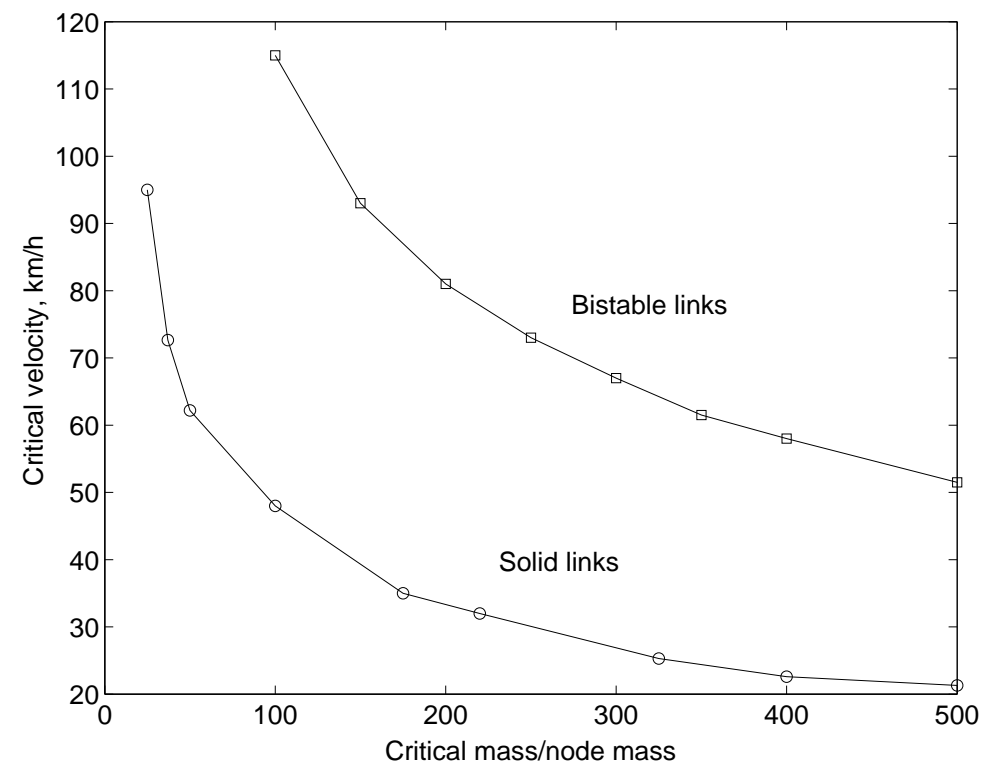

Fig. 13. The mass versus velocity of the projectile that breaks the chain

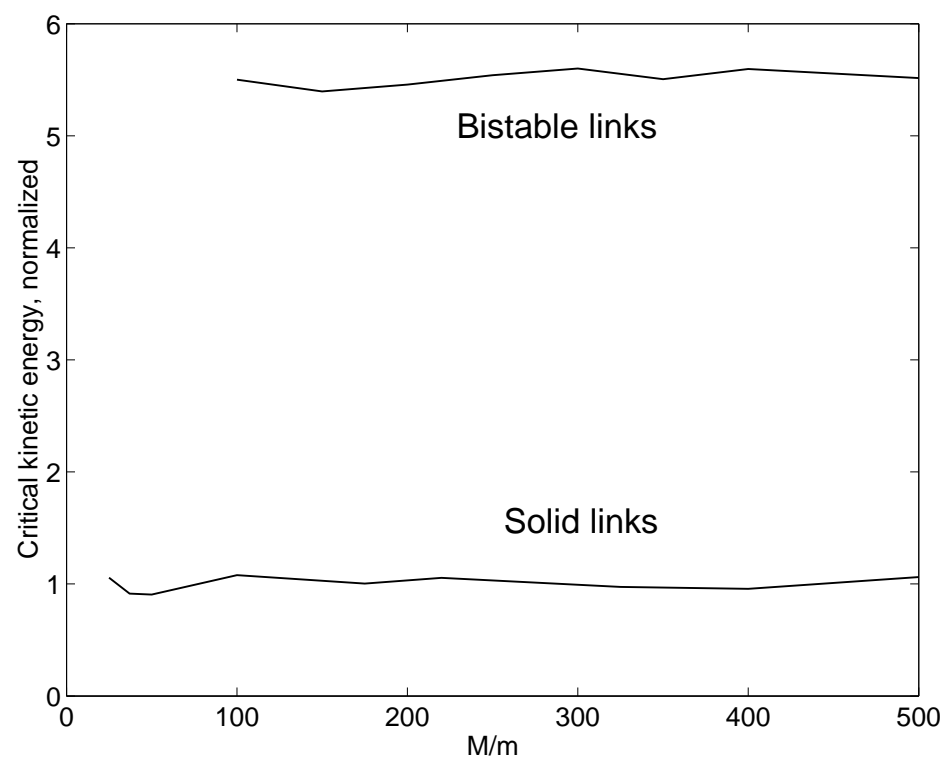

Fig. 14. Kinetic energy of the projectile that breaks the chain

larger mass or velocity breaks it. The velocity of the critical projectile vanishes at the moment when the chain fails and its elongation reaches the maximum. The critical projectile is described by two parameters, mass $M$ and velocity $v_{0}$. These critical parameters are important characteristics of the chain because they determine its capability to resist the impact. Figure 13 compares the critical parameters of the bistable and conventional chains of equal mass, length, and material. Notice that the kinetic energy of the critical projectile stays approximately constant; the bistable chain of 15 links shows five times higher critical energy than a conventional chain, see Figure 14. 


\section{Conclusions}

- The bistable structures can absorb many times more energy than the conventional structures of the same size, mass, and material before failure due to an impact. The amount of the energy needed to break the chain is proportional to its volume not cross-section.

- Damage is not accumulated in a certain location, but is uniformly distributed over the structure. The bistable chains are capable of spreading the damage along the chain.

- The superior impact resistance is achieved by the design of the links that leads to bistability of the effective force-elongation relation. The bistable structures experience the phase transition that is accompanied by an intensive energy consumption.

Acknowledgment. The work was supported by the ARO Grant No: 41363-MA.

\section{References}

[1] Leonid Slepyan, Andrej Cherkaev. Waiting Element Structures and Stability under Extension. Int. J. Damage Mechanics, 1995, 4, No 1, 58-82.

[2] Leonid Slepyan, Andrej Cherkaev, Elena Cherkaev. Transition waves in bistable structures. I. Delocalization of damage. J. Mech. Phys. Solids, 53 (2), 2005, 383405.

[3] Leonid Slepyan, Andrej Cherkaev, Elena Cherkaev. Transition waves in bistable structures. II. Analytical solution: Wave speed and energy dissipation. J. Mech. Phys. Solids, 53 (2), 2005, 407-436.

[4] Leonid Slepyan, Andrej Cherkaev, Elena Cherkaev, Vladimir Vinogradov. Transition waves in controllable cellular structures with high structural resistance. In: W. Gutkowski, T. A. Kowalewski (Eds.), Proceedings of XXI International Congress of Theoretical and Applied Mechanics, Warsaw, Poland 2004, SM24_12170. ISBN 83-89687-01-1.

[5] Andrej Cherkaev, Liya Zhornitskaya. Dynamics of damage in two-dimensional structures with waiting links In: Asymptotics, Singularities and Homogenisation in Problems of Mechanics, A.B.Movchan editor, Kluwer 2004. 273 - 284.

[6] Alexander Balk, Andrej Cherkaev, and Leonid Slepyan. Dynamics of chains with non-monotone stress-strain relations. J. Mech. Phys. Solids, 49 (2001) pp. 131-148.

[7] Alexander Balk, Andrej Cherkaev, and Leonid Slepyan. Nonlinear Waves and Waves of Phase Transition, J. of Phys. Mech. Solids, J. Mech. Phys. Solids. 49 (2001) 149-172.

[8] Leonid Slepyan and Mark Ayzenberg-Stepanenko. Localized transition waves in bistable-bond lattices. J. Mech. Phys. Solids 52 (2004), no. 7, 1447-1479.

[9] Leonid Slepyan. Models and phenomena in fracture mechanics. Foundations of Engineering Mechanics. Springer-Verlag, Berlin, 2002. 
[10] Leonid Slepyan. Feeding and dissipative waves in fracture and phase transition. I. Some 1D structures and a square-cell lattice. J. Mech. Phys. Solids 49 (2001), no. 3, 469-511.

[11] Leonid Slepyan. Feeding and dissipative waves in fracture and phase transition. II. Phase-transition waves. J. Mech. Phys. Solids 49 (2001), no. 3, 513-550.

[12] Leonid Slepyan. Feeding and dissipative waves in fracture and phase transition. III. Triangular-cell lattice. J. Mech. Phys. Solids 49 (2001), no. 12, 2839-2875.

[13] R. Hill. The Mathematical Theory of Plasticity. Oxford Univ Press, 1998.

[14] Akhtar S. Khan, Sujian Huang. Continuum Theory of Plasticity. Wiley, 1995. 